\title{
Title: $\quad$ An Integrator-Differentiator TIA Using a Multi-Element Pseudo- Resistor in its DC Servo Loop for Enhanced Noise Performance
}

Author(s): Haberle, M., Djekic, D., Fantner, G. E., Lips, K., Ortmanns, M., \& Anders, J.

Document type: Postprint

Terms of Use: $\quad$ Copyright applies. A non-exclusive, non-transferable and limited right to use is granted. This document is intended solely for personal, non-commercial use.

Citation:

(C) 2017 IEEE. Personal use of this material is permitted. Permission from IEEE must be obtained for all other uses, in any current or future media, including reprinting/republishing this material for advertising or promotional purposes, creating new collective works, for resale or redistribution to servers or lists, or reuse of any copyrighted component of this work in other works.

Haberle, M., Djekic, D., Fantner, G. E., Lips, K., Ortmanns, M., \& Anders, J. (2018). An Integrator-Differentiator TIA Using a Multi-Element Pseudo-Resistor in its DC Servo Loop for Enhanced Noise Performance. ESSCIRC 2018 - IEEE 44th European Solid State Circuits Conference (FS.SC.IRC.) httns///dni nra/10 1109/esscirc. 0018 8494290 


\title{
An integrator-differentiator TIA using a multi-element pseudo-resistor in its DC servo loop for enhanced noise performance
}

\author{
Matthias Häberle $^{1}$, Denis Djekic ${ }^{2}$, Georg Fantner ${ }^{3}$, Klaus Lips ${ }^{4}$, Maurits Ortmanns ${ }^{1}$, and Jens Anders ${ }^{2}$ \\ Email: matthias.haeberle@uni-ulm.de \\ ${ }^{1}$ Institute of Microelectronics, University of Ulm, D-89081 Ulm, Germany \\ ${ }^{2}$ Institute of Smart Sensors, University of Stuttgart, D-70569 Stuttgart, Germany \\ ${ }^{3}$ Laboratory for Bio- and Nano-Instrumentation, EPFL Lausanne, CH-1015 Lausanne, Switzerland \\ ${ }^{4}$ Berlin Joint EPR Lab, Helmholtz-Zentrum Berlin for Materials and Energy, D-14109 Berlin, Germany
}

\begin{abstract}
In this paper, we present an integratordifferentiator transimpedance amplifier (TIA) featuring a multielement pseudo-resistor (MEPR) in the DC feedback path for improved noise performance in the presence of non-zero DC input currents. The presented prototype is implemented in a standard $180 \mathrm{~nm}$ CMOS technology and achieves an inband transimpedance of $10 \mathrm{M} \Omega$ over a $2.7 \mathrm{MHz}$ signal bandwidth. The MEPR resistor in the DC servo loop can be tuned between $700 \mathrm{k} \Omega$ and $100 \mathrm{M} \Omega$ enabling a precise adjustment of the TIA's lower cutoff frequency. For a DC feedback resistance of $700 \mathrm{k} \Omega$, the TIA provides an input referred noise floor of $180 \mathrm{fA} / \sqrt{\mathrm{Hz}}$ at zero input current, which only marginally increases to $220 \mathrm{fA} / \sqrt{\mathrm{Hz}}$ for the maximum bias current of $1 \mu \mathrm{A}$. The TIA consumes $0.6 \mathrm{~mm}^{2}$ of chip area and $18.5 \mathrm{~mW}$ of power from a $1.8 \mathrm{~V}$ supply.
\end{abstract}

\section{INTRODUCTION}

Several emerging sensing applications in the life and material sciences require precise current measurements with large dynamic ranges, potentially even around large DC input currents. Examples include nanopore sensing [1], scanning ion condcutance microscopy (SICM) [2] and electrically detected magnetic resonance [3]. In addition, these applications frequently require large signal bandwidth to allow for the time-resolved monitoring of processes with improved temporal resolutions in the sub-microsecond range. For such specifications, despite recent advances in resistive TIAs that use active elements in their feedback path, cf. [4], the integratordifferentiator TIA (I-D-TIA) [5], which uses a small integration capacitance in the feedback of the first stage, is generally considered the best choice to achieve an optimal noise bandwidth trade-off. However, state-of-the-art implementations of I-D-TIAs, cf. e.g. [5], struggle with large DC input currents due to an increased noise floor produced by the MOS diodes in weak inversion in the DC servo loop. Therefore, although input referred noise floors in the single digit $\mathrm{fA} / \sqrt{\mathrm{Hz}}$ region have already been achieved with this topology for a zero DC input current, such a performance has not been reported in the presence of significant DC currents at the TIA's input. However, sinking a DC current plays an important role for many of the above named applications, where the current either represents a feedback variable (SICM) or a DC current is required to bias the device under test in the desired operating region (EDMR). To solve this problem, in this paper, we propose an I-D-TIA that uses a multi-element pseudo-resistor (MEPR) as feedback element in the DC servo loop, to greatly improve the TIA's robustness against shot noise at higher DC current levels.

\section{INTEGRATOR-DIFFERENTIATOR TIA}

The TIA presented in this paper is based on the I-D-TIA topology presented by Ferrari et al. in [5], which is shown in Fig. 1. According to Fig. 1, in the Ferrari TIA, the input current is integrated on the feedback capacitor $C_{\mathrm{i}}$ followed by a differentiator stage to produce an overall flat frequency response in the frequency band of interest. In this configuration, any DC input produces a continuous charging or discharging of the feedback capacitor, eventually saturating the output of the integrator opamp. To avoid the saturation problem, in [5], a DC servo loop has been used that prevents any DC input current from charging/discharging $C_{\mathrm{i}}$. This DC servo loop was composed of an analog filter $H(s)$ and a resistor $R_{\mathrm{DC}}$. For low frequencies, $H(s)$ displays an integrator behavior, resulting in a large loop gain at low frequencies. This large loop gain forces the error between the DC input current and the feedback current through $R_{\mathrm{DC}}$ to be small, thereby preventing any net low-frequency current from charging/discharging the feedback capacitor $C_{\mathrm{i}}$. To ensure a sufficiently large phase margin for the feedback loop, $H(s)$ has to contain a zero at an appropriate frequency $f_{\mathrm{z}}$, resulting in a constant attenuation with a factor $\gamma$ immediately following $f_{\mathrm{z}}$, before $H(s)$ eventually rolls off due to parasitic poles. Due to the integrator-differentiator structure combined with the DC servo loop, the Ferrari-TIA features separate outputs for AC signals (passband of the I-D-TIA) and DC/very low frequencies (passband of the DC servo loop) at voltages $\mathrm{V}_{\text {out,AC }}$ and $\mathrm{V}_{\text {out,DC}}$, in Fig. 1.

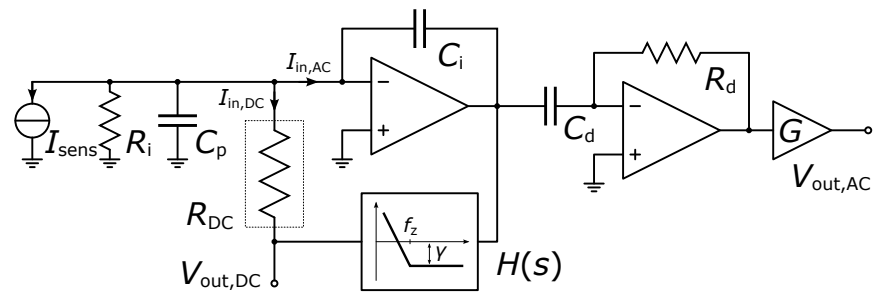

Fig. 1. Illustration of the integrator-differentiator transimpedance (TIA) architecture with time-continuous feedback. 
More specifically, the AC output features a bandpass characteristic with a lower cutoff frequency at:

$$
f_{\mathrm{m}}=\frac{1}{2 \pi C_{\mathrm{i}} R_{\mathrm{DC} \gamma}},
$$

where $C_{\mathrm{i}}$ and $R_{\mathrm{DC}}$ are the integration capacitance and the DC feedback resistor, respectively, and $\gamma$ is the abovementioned constant attenuation factor of $H(s)$ following the zero at $f_{\mathrm{z}}$. To ensure stability, it is mandatory to keep $f_{\mathrm{z}}$ below $f_{\mathrm{m}}$. Above $f_{\mathrm{m}}$, the DC servo loop is essentially inactive and the input signal propagates to the $\mathrm{AC}$ output $\mathrm{V}_{\text {out,AC. }}$. Within the passband of the AC output, $\mathrm{V}_{\text {out,AC }}$, the TIA transimpedance is given by:

$$
Z_{\mathrm{TIA}, \mathrm{AC}}=\frac{R_{\mathrm{d}} C_{\mathrm{d}}}{C_{\mathrm{i}}} \cdot G,
$$

where $C_{\mathrm{i}}$ is the integration capacitance, $R_{\mathrm{d}}$ and $C_{\mathrm{d}}$ are the differentiator resistance and capacitance, respectively, and $G$ is an optional voltage gain following the differentiator, cf. Fig. 1.

In all of the applications listed in Sec. I, the TIA noise is crucial because it directly determines the achievable limit of detection. Here, the main contributers to the TIA noise are the integrator's opamp noise $S_{\Delta \mathrm{V}_{\mathrm{n}, \mathrm{OA}}^{2}}$, which, input referred, is shaped by the feedback capacitance $C_{\mathrm{i}}$ and the parasitic input capacitance $C_{\mathrm{p}}$, cf. Fig. 1, as well as the thermal noise of the resistor $R_{\mathrm{DC}}$. Their resulting input referred current noise PSD, $S_{\Delta \mathrm{I}_{\mathrm{n}, \mathrm{eq}}^{2}}$ is given by:

$$
S_{\Delta \mathrm{I}_{\mathrm{n}, \mathrm{eq}}^{2}}(\omega) \approx \frac{4 k T}{R_{\mathrm{DC}}}+\omega^{2}\left(C_{\mathrm{p}}+C_{\mathrm{i}}\right)^{2} S_{\Delta \mathrm{V}_{\mathrm{n}, \mathrm{OA}}^{2}},
$$

where $k$ is Boltzmann's constant and $T$ is absolute temperature. For higher frequencies, the integrator opamp noise dominates the input referred noise. The opamp's thermal noise can be reduced by increasing the transconductance $G_{\mathrm{m}}$ of its input stage with a trade-off in power consumption. For a given integrator opamp noise $S_{\Delta \mathrm{V}_{\mathrm{n}, \mathrm{OA}}^{2}}$, to minimize its effect on the total input referred noise according to (3), the feedback capacitance $C_{\mathrm{i}}$ should in principle be chosen as small as possible. However, according to (3), a reduction of the value of $C_{\mathrm{i}}$ below that of the parasitic input capacitance $C_{\mathrm{p}}$ is not useful. Another constraint on the minimum useful value of $C_{\mathrm{i}}$ is imposed by the maximum integrator output swing before the onset of intolerable distortion. Here, large distortion first occurs for the smallest inband frequencies, where the integrator transimpedance $1 /\left(\omega C_{\mathrm{i}}\right)$ is the largest. Consequently, as already discussed in [5], the dynamic range of the I-D-TIA is frequency dependent, where the lower end of the AC passband frequency places the hardest constraint on the minimum usable value of $C_{\mathrm{i}}$. While there are many applications, such as classical, mono-frequency lock-in measurements, where the frequency dependent dynamic range is of minor importance, for multi-frequency signals, as they e.g. occur due to the excitation scheme in pulsed EDMR, the frequencydependent clipping of the internal integrator output can present a severe problem, cf. Sec. IV.

The input referred noise floor is defined by the feedback resistor $R_{\mathrm{DC}}$, making it desirable to choose the value of $R_{\mathrm{DC}}$ as large as possible. Here, the upper bound for $R_{\mathrm{DC}}$ is determined by the maximum tolerable voltage swing across $R_{\mathrm{DC}}$ for a given DC input current range, keeping in mind that all of the DC input current flows through $R_{\mathrm{DC}}$, and/or the maximum tolerable chip area for $R_{\mathrm{DC}}$. To mitgate the latter constraint, in [5], a combination of current starving circuits and MOS pseudo-resistor was used. However, as discussed in [4], the MOS pseudo-resistors both display a large PVT dependence, potentially severely degrading the TIAs stability, and an increased shot noise floor when a large DC bias current flows through them. To avoid these problems, in this paper, we propose the use of MEPRs, as introduced in [4] within the ID-TIA, which allows the formation of large resistance values with a greatly improved PVT variation immunity, linearity and noise behavior compared to conventional MOS diode based pseudo-resistors, as was used in [5].

\section{Multi-Element Pseudo-Resistors}

The use of MEPRs to realize large resistance values in the feedback of a resistive TIA was proposed in [4]. As shown in this paper, the use of MEPRs instead of simple MOS pseudoresistors provides several intrinsic advantages: First, thanks to the pseudo current mirror based biasing, the resistance value of the MEPR is very robust against process variations. Next, the MEPR linearity is improved by a series connection of a large number of pseudo-resistors, effectively linearizing the overall I/V-characteristic of the MEPR. Additionally, connecting a large number of elements in series reduces the dependency on device mismatch by averaging, and, therefore further improves the MEPR's robustness against process variations. Finally, the series connection of multiple resistor elements with reduced unit resistance produces an improved $1 / f$ - and shot noise performance compared to a single-element pseudo-resistor, which will be briefly explained in the following.

A long channel MOS transistor with its channel noise being the only noise source under consideration can be modeled as a noiseless transistor with an additive noise current source with the following current noise PSD:

$$
S_{\Delta \mathrm{I}_{\mathrm{nD}}^{2}}=4 k T G_{\mathrm{nD}}
$$

with Boltzmann's constant $k$, absolute temperature $T$, and the channel noise conductance $G_{\mathrm{nD}}$. In case of operating deeply in the linear region, i. e., for $V_{\mathrm{DS}} \approx 0$, the inverse channel noise conductance is approximately equal to the channel resistance and, therefore, the transistor displays the same thermal noise as an equivalent ohmic resistor. Additionally, a chain of noisy resistors shows the same noise behavior as a single resistor of the total resistance value. Therefore, the noise contribution to the TIA's equivalent input noise PSD of a MEPR in the linear mode $\left(I_{\mathrm{DC}}=0\right)$ with a total value of $R_{\mathrm{DC}}$ is:

$$
S_{\Delta \mathrm{I}_{\mathrm{n}, \mathrm{eq}}^{2}}=4 k T / R_{\mathrm{DC}} \text {. }
$$

However, when a non-zero current is flowing, i.e. $I_{\mathrm{DC}}>0$, the thermal noise of the pseudo-resistor can be dominated by shot noise. In this case, the noise current PSD of a single MOS transistor becomes:

$$
S_{\Delta \mathrm{I}_{\mathrm{nD}}^{2}}=2 q I_{\mathrm{DC}}
$$

where $q$ is the elementary charge. In addition to an increased noise floor due to shot noise, especially at low frequencies and elevated DC input currents, MOS based pseudo-resistors can display significant $1 / f$-noise. Since both the $1 / f$ - and the shot noise components of a MOS pseudo-resistor can easily exceed the thermal noise limit of (5), its intrinsic 


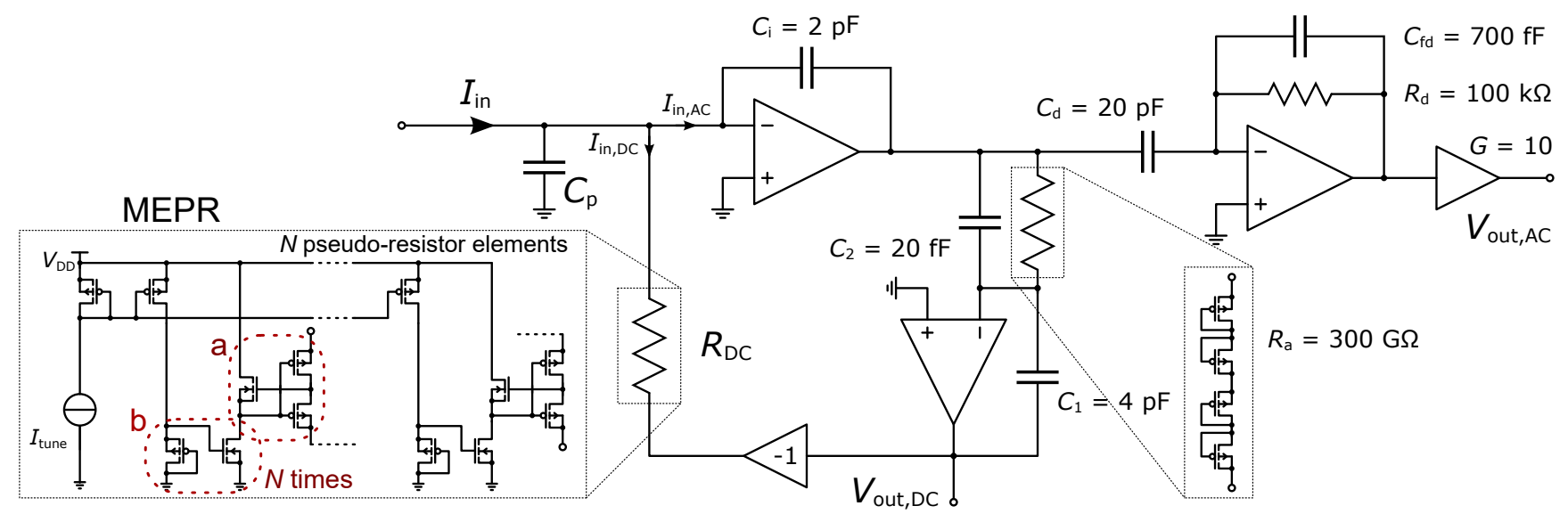

Fig. 2. Implemented integrator-differentiator TIA with tunable $R_{D C}$, realized as MEPR using a: tunable pseudo-resistors; b: pseudo current mirror. $R_{a}$ is implemented as a conventional pseudo-resistor using PMOS diodes.

suppression of both noise sources renders the MEPR very suitable for precision current sensing applications with nonzero DC current components. More specifically, in [4], it has been shown, that for an $N$-element MEPR, consisting of $2 N$ noisy transistors, due to the current divider structure of the MEPR, the total input referred noise PSD is reduced compared to that of a single MEPR transistor by the number of MEPR elements according to:

$$
S_{\Delta \mathrm{I}_{\mathrm{n}, \mathrm{eq}, \mathrm{tot}}^{2}}=\frac{S_{\Delta I_{\mathrm{nD}}^{2}}}{2 N} .
$$

For a chain of $2 N$ ohmic resistors (and therefore also for MOS pseudo-resistors in the linear region) with a fixed total resistance $R_{\text {tot }}=2 N R_{\text {unit }}$, (7) describes the intuitive fact that the total input referred noise is independent of the number of unit elements and solely depends on the total resistance value. However, if the noise floor of each unit element exceeds the thermal noise floor, the MEPR effectively suppresses this excess noise, producing a total input referred noise close to that of an ohmic resistor corresponding to the total MEPR resistance, cf. [4]. It is this suppression of shot noise and $1 / f$-noise that makes the MEPR particularly suitable for the realization of the feedback resistor $R_{\mathrm{DC}}$ in the DC servo loop of Fig. 1.

\section{Prototype Realization and Measurements}

A prototype of the proposed I-D-TIA using a MEPR in the DC servo loop was implemented in a standard $180 \mathrm{~nm}$ CMOS technology. Here, in contrast to the design in [4], a standard CMOS process was sufficient because the parasitic well capacitance of the MEPR is not critical in the DC servo loop. To achieve a passband transimpedance of $10 \mathrm{M} \Omega$ over a bandwidth of $2.7 \mathrm{MHz}$, the TIA circuit components were chosen with the aid of (2), cf. Fig. 2. Here, according to the discussion of Sec. II, a compromise between input noise and integrator output swing has been accomplished by implementing a feedback capacitance of $C_{\mathrm{i}}=2 \mathrm{pF}$, which is approximately equal to the total estimated parasitic input capacitance. Since $C_{\mathrm{i}}$ is approximately equal to $C_{\mathrm{p}}$, the input referred noise $S_{\Delta \mathrm{I}_{\mathrm{n}, \text { eq }}^{2}}$ is increased by an acceptable amount while the integrator's dynamic range is enhanced by a factor of approximately 20 compared to [5], which used $C_{\mathrm{i}}=100 \mathrm{fF}$.

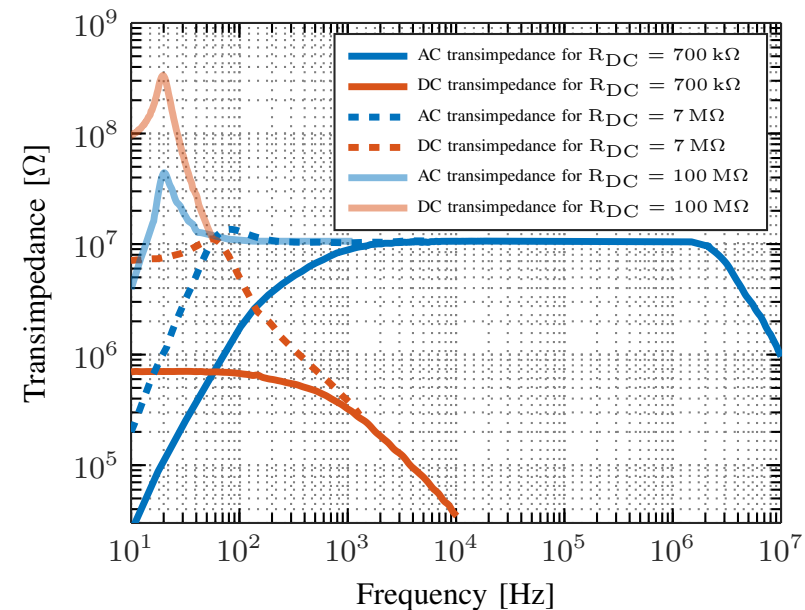

Fig. 3. Measured transimpedances of the TIA's AC and DC outputs for different MEPR values. The biasing current is set to $I_{\text {tune }}=90 \mu \mathrm{A}, I_{\text {tune }}=$ $5 \mu \mathrm{A}$, and $I_{\text {tune }}=400 \mathrm{nA}$ in order achieve a feedback resistance of $R_{\mathrm{DC}}=$ $700 \mathrm{k} \Omega, R_{\mathrm{DC}}=7 \mathrm{M} \Omega$, and $R_{\mathrm{DC}}=100 \mathrm{M} \Omega$, respectively.

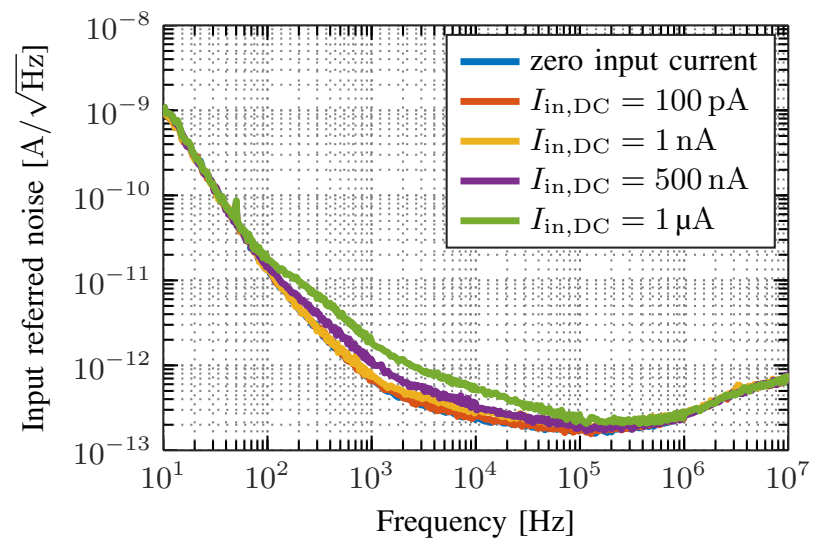

Fig. 4. Input referred noise current density for various DC currents

According to Sec. II the zero at $\mathrm{f}_{\mathrm{z}}$ formed by $R_{\mathrm{a}}$ and $C_{2}$ needs to satisfy the condition $f_{\mathrm{z}}<f_{\mathrm{m}}$ to ensure stability. Therefore, $H(s)$ has been implemented with $R_{\mathrm{a}}$ as a conventional pseudo-resistor based on PMOS diodes (simulated resistance $300 \mathrm{G} \Omega$ ) and an attenuation of $\gamma=\frac{C_{1}}{C_{2}}=200$, cf. Fig. (2). Since the conventional pseudo-resistor $R_{\mathrm{a}}$ is prone to process 


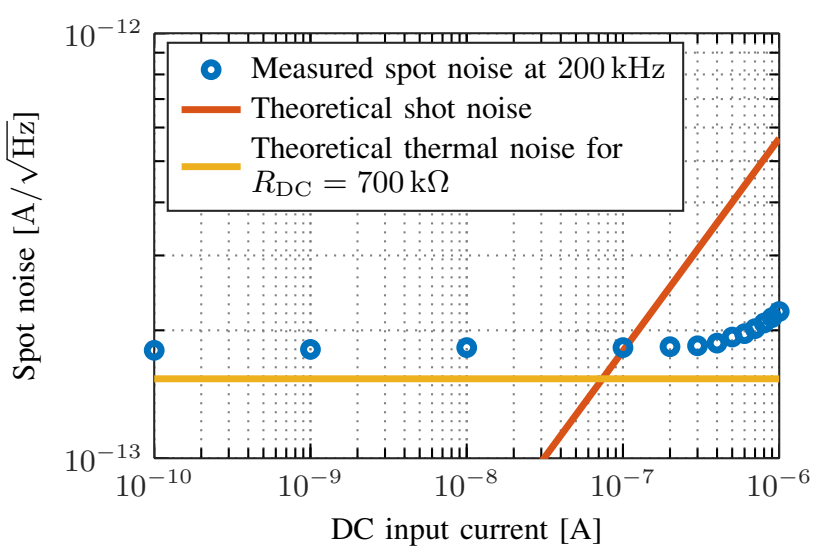

Fig. 5. Measured input referred spot noise at $200 \mathrm{kHz}$ vs. DC curent.

variations, it has been over-designed to garantuee stability over process corners. The MEPR is implemented using 16 elements because, as derived in [4], this choice presents a good compromise between linearity, area and maximum achievable output swing. For maximum flexibility, the biasing current $I_{\text {tune }}$, which can be used to fine tune the resistance of the MEPR, is generated off-chip using a programmable external current source (Keithley 6221). Fig. 3 shows the respective frequency responses of the TIA outputs for various feedback resistances $R_{\mathrm{DC}}$. Fig. 3 illustrates the effect of the value of $R_{\mathrm{DC}}$ on the lower cutoff frequency of the AC passband, $f_{\mathrm{m}}$, and the phase margin of the feedback loop. The maximum useful value of the MEPR is reached somewhere below $R_{\mathrm{DC}}=100 \mathrm{M} \Omega$, since at $R_{\mathrm{DC}}=100 \mathrm{M} \Omega$ already significant peaking around $12 \mathrm{~dB}$ occurs, due to the reduced phase margin, as $f_{\mathrm{m}}$ approaches $f_{\mathrm{z}}$. Fig. 4 shows the input referred noise for a feedback resistance of $R_{\mathrm{DC}}=700 \mathrm{k} \Omega$ and different DC input currents $I_{\mathrm{in}, \mathrm{DC}}$ including $I_{\mathrm{in}, \mathrm{DC}}=0 \mathrm{~A}$. The input referred noise was obtained by measuring the noise at the $\mathrm{AC}$ output and dividing by the AC transimpedance of Fig. 3 . According to Fig. 4, the white noise floor increases only very little for DC inputs of $100 \mathrm{pA}$ and $1 \mathrm{nA}$ compared to the zero input case. Even for the maximum input current of $1 \mu \mathrm{A}$, the lower white noise floor increases by less than a factor of two. As an example of a conventional pseudo-resistor feedback - although at different absolute noise and DC input current levels -, in [5], the current increased more than 20-fold for the maximum DC input current. This clearly demonstrates the improved shot noise immunity of the proposed MEPR based DC servo loop compared to the one proposed in [5]. As expected, the high frequency input noise is unaffected by the input DC current, since it is solely determined by the shaped opamp noise $S_{\Delta \mathrm{V}_{\mathrm{n}, \mathrm{OA}}^{2}}$, cf. (3). To further validate the theoretically predicted immunity of the MEPR against an increased noise floor due to shot noise, the input referred noise floor at $200 \mathrm{kHz}$ has been measured in more detail as a function of the DC input current. The corresponding results are shown in Fig. 5, where they are graphically compared against the theoretical thermal noise limit of an equal size ohmic resistor of $R_{\mathrm{DC}}=700 \mathrm{k} \Omega$, as well as the theoretical shot noise value of a single-element pseudo-resistor, cf. (5). Here, the largest DC current of $1 \mu \mathrm{A}$, was limited by the maximum voltage swing of VDD/2 across the MEPR: According to Fig. 5, shot noise would dominate a single-element pseudo-resistor for DC currents $>100 \mathrm{nA}$. However, due to the intrinsic shot noise suppression of the MEPR, the measured noise floor of the

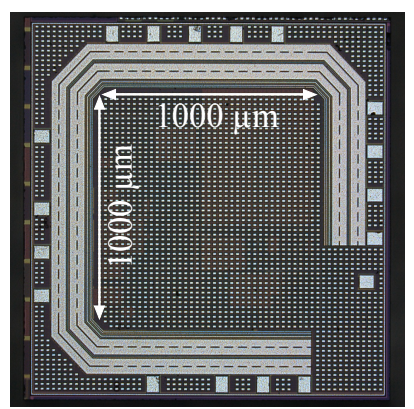

Fig. 6. Die micrograph. The TIA consumes $0.6 \mathrm{~mm}^{2}$ of chip area.

presented TIA stays well below the theoretical line for a singleelement pseudo-resistor, reaching a maximum of $220 \mathrm{fA} / \sqrt{\mathrm{Hz}}$ for a bias current of $1 \mu \mathrm{A}$. This result clearly verifies the superior shot noise performance of the MEPR compared to a single-element pseudo-resistor.

\section{CONClusion}

In this paper, an I-D-TIA featuring a MEPR-based DC servo loop for an improved noise performance at non-zero DC currents has been presented. The TIA was manufactured in a standard $180 \mathrm{~nm}$ CMOS technology and achieves an inband transimpedance of $10 \mathrm{M} \Omega$ over a bandwidth of $2.7 \mathrm{MHz}$ and an input referred noise floor between $180 \mathrm{fA} / \sqrt{\mathrm{Hz}}$ (for $\left.I_{\mathrm{in}, \mathrm{DC}}=0 \mathrm{~A}\right)$ and $220 \mathrm{fA} / \sqrt{\mathrm{Hz}}\left(\right.$ for $\left.I_{\mathrm{in}, \mathrm{DC}}=1 \mu \mathrm{A}\right)$. Therefore, the measured noise floor, even for the largest DC current is within a factor of two of the theoretical limit of an ohmic DC feedback resistor of equal size. Thanks to the relatively large integration capacitance of $C_{\mathrm{i}}=2 \mathrm{pF}$ in combination with the MEPR feedback resistor, the proposed design features an approximately 20 -fold increased dynamic range vs. frequency compared to previous integrator-differentiator TIA [5] together with a significantly reduced increase in its noise floor at larger DC input currents. Overall, the proposed TIA provides a performance, that makes it a very promising candidate for many emerging biomedical and materials science applications, which require precise current measurements around non-zero DC currents and with large dynamic ranges.

\section{ACKNOWLEDGMENT}

This work was supported by the DFG in the frame of GRK2203 (PULMOSENS).

\section{REFERENCES}

[1] J. K. Rosenstein et al., "Integrated nanopore sensing platform with submicrosecond temporal resolution," Nature Methods, vol. 9, no. 5, pp. 487-492, May 2012.

[2] P. Novak et al., "Nanoscale live cell imaging using hopping probe ion conductance microscopy," Nature Methods, vol. 6, no. 4, pp. 279-281, Apr 2009.

[3] J. M. Elzerman et al., "Single-shot read-out of an individual electron spin in a quantum dot," Nature, vol. 430, no. 6998, pp. 431-435, Jul 2004.

[4] D. Djekic et al., "A transimpedance amplifier using a widely tunable pvt-independent pseudo-resistor for high-performance current sensing applications," in ESSCIRC 2017 - 43rd IEEE European Solid State Circuits Conference, Sept 2017, pp. 79-82.

[5] G. Ferrari et al., "Transimpedance amplifier for high sensitivity current measurements on nanodevices," IEEE Journal of Solid-State Circuits, vol. 44, no. 5, pp. 1609-1616, May 2009. 